\title{
Assessment of the SRC Inhibition Role in the Efficacy of Breast Cancer Radiotherapy
}

\author{
Shabnam Shahrokh', Vahid Mansouri ${ }^{2}$, Mohammadreza Razzaghi ${ }^{3}$ \\ ${ }^{1}$ Gastroenterology and Liver Diseases Research Center, Research Institute for Gastroenterology and Liver Diseases, Shahid \\ Beheshti University of Medical Sciences, Tehran, Iran \\ ${ }^{2}$ Proteomics Research Center, Faculty of Paramedical Sciences, Shahid Beheshti University of Medical Sciences, Tehran, \\ Iran \\ ${ }^{3}$ Laser Application in Medical Sciences Research Center, Shahid Beheshti University of Medical Sciences, Tehran, Iran
}

\section{*Correspondence to \\ Vahid Mansouri, Proteomics \\ Research Center (PRC), Darband St. \\ Qods Sq., Tehran, Iran. \\ Tel: +982122714248 \\ Email:vm1343@yahoo.com}

Published online December 1, 2019

\begin{abstract}
Introduction: Radiotherapy (RT) is an effective therapeutic method for preventing the recurrence of breast cancer after surgery. The development and improvement of RT are of interest to scientists. Possible improvement of RT via study of dysregulated proteins of breast cancer cell line MDA-MB-231 which is exposed to 10 Gray (Gy) radiation is aim of this study.

Methods: Using protein-protein interaction (PPI) network analysis by means of running Cytoscape software via the STRING database, the up-regulated proteins of MDA-MB-231 breast cancer cells irradiated by a single and fractioned 10 Gy $137 \mathrm{Cs} \gamma$-radiation were analyzed. The network was analyzed by using the Network analyzer to characterize the central genes. The action map was mapped for the queried genes and the added neighbors via CluePedia-STRING ACTIONS-v10.5- 20.11.2017.

Results: The 14 differentially expressed proteins (DEPs) plus 10 neighbors interacted to construct a network. Among the 14 queried DEPs, FN1, CSPG4, LRP1, GSN, RTN4, and CTSD were highlighted as a complex set in the analysis. The analysis revealed that SRC as an added neighbor was activated by the critical DEPs. The activation of other oncogenes like AKT1 was also determined.

Conclusion: The results indicate that the inhibition of SRC activity or the inhibition of its activators is a useful function of breast cancer RT.

Keywords: Breast cancer; Cell; Gene; Radiation; Network.
\end{abstract}

\section{Introduction}

Radiotherapy (RT) after a mastectomy is an effective tool for preventing a recurrence of breast cancer. ${ }^{1}$ Investigations indicate that the addition of chemotherapy to RT improves the efficiency of treatment in cancer patients. ${ }^{2}$ There are serious efforts to improve and develop RT efficacy. ${ }^{3}$ It is reported that the inhibition of certain signaling pathways is associated with the enhancement of RT response in several cancer types. ${ }^{4,5}$

Proteomics is a large-scale method for determining the changed expression proteins after intervention in the samples. In this method, large numbers of proteins are introduced, which are involved in response to the interpolation. ${ }^{6}$ Proteomic investigations have attracted the attention of many scientists in medicine, especially in cancer therapy. Clinical proteomics plays a significant role in managing cancer in patients. As it is reported, based on proteomic findings, breast cancer classification is affected by proteomic investigation. ${ }^{7,8}$ Besides proteomics, bioinformatics affects clinical research, especially in the field of cancer. ${ }^{9}$

Network analysis is a method that provides valuable data which are useful to screen the analyzed and ranked subjects based on interactions between the members of a sample. ${ }^{10,11}$ Protein-protein interaction (PPI) network analysis is used to interpret the molecular mechanism of many diseases such as different types of cancers, neurodegenerative diseases, and metabolic disorders in medicine. ${ }^{12-14}$ Laser therapy is evaluated in skin samples by PPI network analysis and the cytokine-mediated signaling pathway is introduced as a critically affected pathway in the patients. ${ }^{15}$ In the present study, the differentially expressed proteins (DEPs) associated with radiation in MDA-MB-231 breast cancer cells are investigated to find a possible developmental way for the RT of breast cancer.

\section{Methods}

Following the procedure performed by et al, ${ }^{16}$ we exposed 
MDA-MB-231 breast cancer cells to a single and fractioned 10 Gray (Gy) 137Cs $\gamma$-radiation (Gammacell 3000 Elan, MDS Nordion, Canada) and harvested them after 48 hours. In this investigation, the up-regulated proteins which were common between the single and fractioned irradiated cells were determined. The queried DEPs were included in the PPI network to form interactome by Cytoscape software v6.3.1 via the STRING database. ${ }^{17}$ Due to increased connections between the queried DEPs, 10 neighbors from the STRING database were added to the DEPs and a new network was created. The network was analyzed by the network analyzer. The descriptions of the 14 queried DEPs were extracted from the STRING database $^{18}$ and were summarized. Since the action map provides useful data about the elements of a network, binding, activation, inhibition, catalysis, expression, and ptmod actions were mapped for the 24 proteins via CluePedia-STRING ACTIONS-v10.5- 20.11.2017. ${ }^{19}$

\section{Results}

The 14 DEPs were included in the PPI network and the nodes were connected via undirected edges (see Figure 1). The network included 4 isolated nodes, four paired proteins and a main connected component. There were not only 4 isolated nodes but also poor connections between the elements of the network. Due to poor connections between the nodes of the constructed network and also making linkages between the isolated individuals, another network including the added relevant neighbors was created. The newly created network contained 14 queried DEPs plus 10 add neighbors. This network is shown in Figure 2. All nodes are connected by undirected edges and centrality parameters (including degree, betweenness centrality, closeness centrality and stress), for each node is different from the others. Descriptions and centrality parameters of the queried DEPs were extracted from the STRING database and are summarized in Table 1. The action map for the $14 \mathrm{DEPs}$ and 10 added neighbors is illustrated in Figure 3.

\section{Discussion}

Proteomics has attracted the attention of many researchers in medicine. Follow up of patients, the discovery of

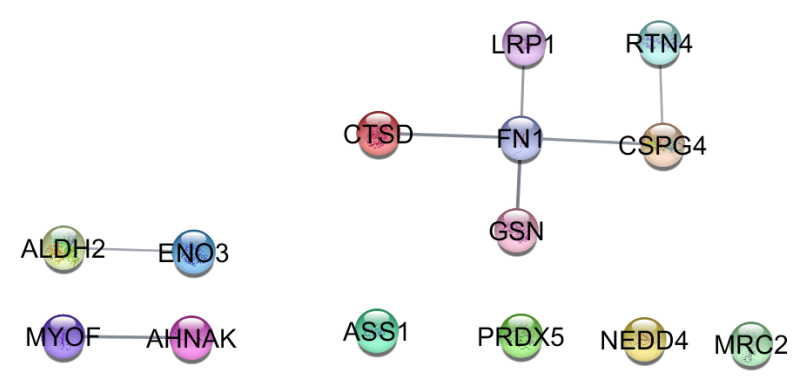

Figure 1. The 14 queried proteins are included in the network. There are poor connections between the genes. diagnostic biomarkers, and the identification of drug targets are the fields that are significantly affected by proteomic investigations. The system biology approach is applied to interpret complex findings about diseases. In this study, 14 identified proteins which were up-regulated in the breast cancer cells under radiation were a candidate for understanding the mechanism of biological effects and screening the studied DEPs to find possible biomarkers. As it is shown in Figure 1, there are a limited number of connections between DEPs that refer to the incomplete information about them in data banks. Adding first 10 neighbors to the DEPs led to constructing an interacted network (see Figure 2).

As it is depicted in Figure 1, MYOF is connected to AHNAK via a physical linkage and both the paired DEPs are isolated from the other nodes. This connection is confirmed in Figure 3 by a binding type of connection between MYOF and AHNAK. AHNAK is connected to AKT1 via a post-translation modification link and whit a similar pattern, NEDD4 is linked to EGFR (see Figure 3). The other paired DEPs are ALDH2 and eno3 that are characterized by no actions in the action map. MRC2 and ASS1 are not only isolated but also disconnected in the action map (see Figure 1).

It seems that a complex including 6 DEPs in Figure 1 is a major player in the effect of radiation on the studied cells. This complex includes FN1, CSPG4, LRP1, GSN, RTN4, and CTSD DEPs. As it is shown in the action map, RTN4 and CTSD are isolated nodes and are not involved in the regulation of the other DEPs. Therefore, it is suggested that FN1, CSPG4, GSN, and LRP1 remain as the critical DEPs which are involved in the effect of the applied intervention. As represented in Table 1, the degree values of FN1, LRP1, GSN, and CSPG4 are 14, 8, 8, and 4

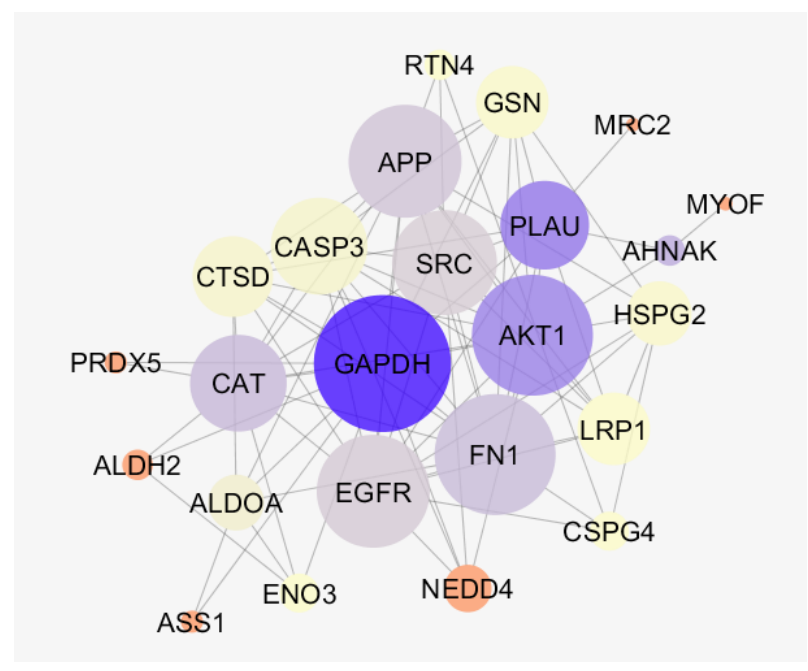

Figure 2. The network constructed from 14 queried proteins plus 10 neighbors. The genes are linked by 90 edges. The bigger size of nodes refers to higher degree value. Colors from yellow to blue consist of the increment of betweenness centrality. 
Table 1. The Summarized Description and Centrality Parameters of 14 Queried DEPs From the STRING Database

\begin{tabular}{|c|c|c|c|c|c|}
\hline Name & Description & K & BC & $\mathrm{CC}$ & Stress \\
\hline FN1 & Fibronectin type III domain containing; endogenous ligands & 14 & 0.07 & 0.7 & 156 \\
\hline CTSD & $\begin{array}{l}\text { Cathepsin D; Acid protease active in intracellular protein breakdown. It plays a role in APP processing } \\
\text { following cleavage and activation by ADAM } 30 \text { which leads to APP degradation. It is involved in the } \\
\text { pathogenesis of several diseases such as breast cancer and possibly Alzheimer disease; cathepsins }\end{array}$ & 9 & 0.01 & 0.61 & 32 \\
\hline GSN & $\begin{array}{l}\text { Actin-depolymerizing factor; calcium-regulated, actin-modulating protein that binds to the plus (or barbed) } \\
\text { ends of actin monomers or filaments, preventing monomer exchange (end-blocking or capping). It can } \\
\text { promote the assembly of monomers into filaments (nucleation) as well as sever filaments already formed. It } \\
\text { plays a role in ciliogenesis; gelsolin/villins }\end{array}$ & 8 & 0.01 & 0.59 & 26 \\
\hline
\end{tabular}

Prolow-density lipoprotein receptor-related protein 1; Endocytic receptor; it is involved in endocytosis and phagocytosis of apoptotic cells, cellular lipid homeostasis, plasma clearance of chylomicron remnants and activated LRPAP1 (alpha 2-macroglobulin), as well as the local metabolism of complexes between plasminogen activators and their endogenous inhibitors. It is required for early embryonic development. It may modulate cellular events, such as APP metabolism, kinase-dependent intracellular signaling, neuronal calcium signaling as well as neurotransmission.

Neural precursor cell expressed, developmentally down-regulated 4, E3 ubiquitin protein ligase; It is involved in the pathway leading to the degradation of VEGFR-2/KDFR, independent of its ubiquitin-ligase activity. Ubiquitinates FGFR1, leading to receptor internalization and degradation in lysosomes. The direct

NEDD4 link between NEDD4 and PTEN regulation through polyubiquitination is questionable. It is involved in the ubiquitination of ERBB4 intracellular domain E4ICD. It is involved in the budding of many viruses. It is part of a signaling complex that regulates neuronal dendrite extension and arborization during development. It ubiquitinates TNK2 and regulates EGF-induced degradation of EGFR and TNF2.

Melanoma-associated chondroitin sulfate proteoglycan; Proteoglycan plays a role in cell proliferation and migration which stimulates endothelial cells motility during microvascular morphogenesis. It may also inhibit neurite outgrowth and growth cone collapse during axon regeneration. Cell surface receptor for collagen alpha 2(VI) which may confer the ability of cells to migrate on that substrate. It may regulate MPP16dependent degradation and invasion of type I collagen participating in melanoma cell invasion properties and modulate the plasminogen system, cell polarization, stimulate alpha-4, beta-1 integrin-mediated adhesion and spreading by recruiting and activating a signaling cascade, and activate FAK and ERK1/ERK2 signaling cascades.

ENO3 2-phospho-D-glycerate hydro-lyase; it appears to have a function in striated muscle development and regeneration; it belongs to the enolase family.

Neuroblast differentiation-associated protein AHNAK; it may be required for neuronal cell differentiation; PDZ domain-containing

Neuroendocrine-specific protein C homolog; Developmental neurite growth regulatory factor with a role as a negative regulator of axon-axon adhesion and growth, and as a facilitator of neurite branching. It is involved in the down-regulation of growth, stabilization of wiring and restriction of plasticity in the adult CNS. It induces the formation and stabilization of endoplasmic reticulum (ER) tubules. It regulates membrane morphogenesis in the ER. It influences NE expansion, nuclear pore complex formation and proper localization of inner nuclear membrane proteins.

ALDH2 Aldehyde dehydrogenase 2 family member; it belongs to the aldehyde dehydrogenase family.

Argininosuccinate synthase 1 ; it is one of the enzymes of the urea cycle, the metabolic pathway transforming neurotoxic ammonia produced by protein catabolism into innocuous urea in the liver of ureotelic animals. It catalyzes the formation of arginosuccinate from aspartate, citrulline and ATP and together with ASL, it is responsible for the biosynthesis of arginine in most body tissues.

Peroxiredoxin-5, mitochondrial; Thiol-specific peroxidase that catalyzes the reduction of hydrogen peroxide and organic hydroperoxides to water and alcohols respectively. It plays a role in cell protection against oxidative stress.

Urokinase-type plasminogen activator receptor-associated protein; it may play a role as an endocytotic lectin receptor displaying calcium-dependent lectin activity. It internalizes glycosylated ligands from the extracellular space for release in an endosomal compartment. It may be involved in the plasminogen activation system, and thus it may regulate protease activity at the cell surface. It may contribute to cellular uptake, remodeling and degradation of extracellular collagen matrices. It may play a role during cancer progression as well as in other chronic tissue destructive diseases acting on collagen turnover.

Fer-1-like protein 3; Calcium/phospholipid-binding protein that plays a role in the plasmalemma repair mechanism of endothelial cells that permit rapid resealing of membranes disrupted by mechanical stress. It is involved in endocytic recycling. It is implicated in VEGF signal transduction by regulating the levels of the receptor KDR (By similarity); Ferlin family

Abbreviations: BC, betweenness centrality; CC, closeness centrality. 


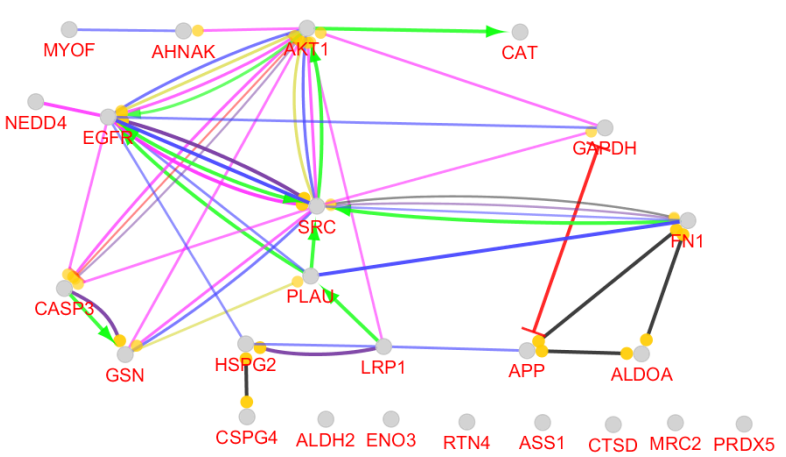

Figure 3. Binding (blue), activation (green), inhibition (red), catalysis (purple), reaction (black), expression (yellow), and ptmod (pink) actions for the 14 queried DEPs plus the 10 added neighbors. Map is illustrated via CluePedia-STRING ACTIONS-v10.5- 20.11.2017.

respectively. These four highlighted DEPs are connected to each other in the action map indirectly.

SRC as a neighbor node plays a critical role in the action map. Various types of connections are issued from SRC to the other genes including the added neighbors or the DEPs. FN1 and GSN are linked to SRC directly in the action map. FN1 activates SRC besides other actions. SRC is an activator for AKT1 and EGFR directly and CAT indirectly. There is another activation path from LRP1PLAU-SRC that refers to the activation of SRC by LRP1 in an indirect path.

There are strong pieces of evidence about the elevation of the SRC level in the body and cancer onset and promotion. $^{20,21}$ This fact implies that SRC is a suitable drug target in cancer treatment. SRC family kinases are introduced as the target in the case of cancer therapy. ${ }^{22}$ Investigations indicate that SRC and also EGFR family overexpress frequently in human cancer. ${ }^{23} \mathrm{~A}$ mutual activation connection between SRC and EGFR is illustrated in the action map. The other activated gene is AKT1. The significant role of AKT1 in cancer development leads to introducing this gene as a drug target in cancer treatment. The inhibition of AKT1 activity has effectively produced therapeutic effects on breast cancer. ${ }^{24,25}$

As it is shown in Figure 3, there is another activation path that starts from LRP1 and affects PLAU and finally ends with EGFR. Therefore, LRP1 activates EGFR indirectly besides activating SRC. The up-regulation of EGFR in many types of cancers along with its role in promoting the growth of solid tumors has been confirmed. It is reported that the detection of the EGFR level is a useful tool for making the prognosis of several cancers. ${ }^{26}$

Gelsolin is the DEP that appears in the described complex in Figure 1. As it is illustrated in Figure 3, GSN is activated in the action map by CASP3. It is reported that GSN RNA production down-regulates in breast cancer of humans and some animals. ${ }^{27}$ The activation of GSN in the action map may be a protecting process against cancer in the treated cancer cells.

\section{Conclusion}

Findings from this study indicate that SRC activation is the main process that occurs in the breast cancer cells treated with irradiation. It can be concluded that the inhibition of positive regulators of SRC is a useful task in breast cancer RT.

\section{Ethical Considerations}

Not applicable.

\section{Conflict of Interests}

The authors declare no conflict of interest.

\section{Acknowledgment}

Shahid Beheshti University of Medical Sciences supports this research.

\section{References}

1. Overgaard M, Jensen MB, Overgaard J, Hansen PS, Rose C, Andersson M, et al. Postoperative radiotherapy in highrisk postmenopausal breast-cancer patients given adjuvant tamoxifen: Danish Breast Cancer Cooperative Group DBCG 82c randomised trial. Lancet. 1999;353(9165):16418. doi: 10.1016/S0140-6736(98)09201-0.

2. Miller ED, Fisher JL, Haglund KE, Grecula JC, Xu-Welliver M, Bertino EM, et al. The addition of chemotherapy to radiation therapy improves survival in elderly patients with stage III non-small cell lung cancer. J Thorac Oncol. 2018;13(3):426-35. doi: 10.1016/j.jtho.2017.11.135.

3. Kang J, Ning MS, Feng H, Li H, Bahig H, Brooks ED, et al. Predicting 5-Year Progression and Survival Outcomes for Early-Stage Non-Small Cell Lung Cancer Treated with Stereotactic Ablative Radiotherapy: Development and Validation of Robust Prognostic Nomograms. Int J Radiat Oncol Biol Phys. 2019. doi: 10.1016/j.ijrobp.2019.09.037.

4. Bhatia S, Sharma J, Bukkapatnam S, Oweida A, Lennon S, Phan A, et al. Inhibition of EphB4-Ephrin-B2 Signaling Enhances Response to Cetuximab-Radiation Therapy in Head and Neck Cancers. Clin Cancer Res. 2018;24(18):453950. doi: 10.1158/1078-0432.CCR-18-0327.

5. Oing C, Tennstedt P, Simon R, Volquardsen J, Borgmann $\mathrm{K}$, Bokemeyer $\mathrm{C}$, et al. BCL2-overexpressing prostate cancer cells rely on PARP1-dependent end-joining and are sensitive to combined PARP inhibitor and radiation therapy. Cancer Lett. 2018;423:60-70. doi: 10.1016/j. canlet.2018.03.007.

6. Jamet E, Santoni V. Editorial for Special Issue: 2017 Plant Proteomics. Proteomes.2018;6(3):E28. doi: 10.3390/ proteomes6030028.

7. Coscia F, Lengyel E, Duraiswamy J, Ashcroft B, BassaniSternberg M, Wierer M, et al. Multi-level proteomics identifies CT45 as a chemosensitivity mediator and immunotherapy target in ovarian cancer. Cell. 2018;175(1):159-70. doi: 10.1016/j.cell.2018.08.065.

8. Yanovich G, Agmon H, Harel M, Sonnenblick A, Peretz T, Geiger T. Clinical proteomics of breast cancer reveals a novel layer of breast cancer classification. Cancer Res. 
2018;78(20):6001-10. doi: 10.1158/0008-5472.CAN-181079.

9. Tyanova S, Cox J. Perseus: a bioinformatics platform for integrative analysis of proteomics data in cancer research. Methods Mol Biol. 2018;1711:133-48. doi: 10.1007/978-14939-7493-1_7.

10. Zhang B, Horvath S. A general framework for weighted gene co-expression network analysis. Statistical applications in genetics and molecular biology. Stat Appl Genet Mol Biol. 2005;4(1). doi: 10.2202/1544-6115.1128.

11. Khanin R, Wit E. How scale-free are biological networks. J Comput Biol. 2006;13(3):810-8. doi: 10.1089/ cmb.2006.13.810.

12. Zamanian-Azodi M, Rezaei-Tavirani M, Rahmati-Rad S, Hasanzadeh H, Tavirani MR, Seyyedi SS. Protein-Protein Interaction Network could reveal the relationship between the breast and colon cancer. Gastroenterol Hepatol Bed Bench. 2015;8(3):215-24. doi: 10.22037/ghfbb.v8i3.763.

13. Rezaei-Tavirani M, Zamanian-Azodi M, Rajabi S, Masoudi-Nejad A, Rostami-Nejad M, Rahmatirad S. Protein Clustering and Interactome Analysis in Parkinson and Alzheimer's Diseases. Arch Iran Med. 2016;19(2):1019. doi: 0161902/AIM.007.

14. Safaei A, Tavirani MR, Azodi MZ, Lashay A, Mohammadi SF, Broumand MG, et al. Diabetic retinopathy and laser therapy in rats: A protein-protein interaction network analysis. J Lasers Med Sci. 2017;8(1):S20-S21. doi: 10.15171/ jlms.2017.s4.

15. Rostami-Nejad M, Rezaei-Tavirani M, Zadeh-Esmaeel MM, Rezaei-Tavirani S, Akbari Z, Esmaeili S, et al. Assessment of cytokine-mediated signaling pathway dysregulation in arm skin after CO2 laser therapy. J Lasers Med Sci.2019;10(4):257-63. doi: 10.15171/jlms.2019.42.

16. Kim MH, Jung SY, Ahn J, Hwang SG, Woo HJ, An S, et al. Quantitative proteomic analysis of single or fractionated radiation-induced proteins in human breast cancer MDAMB-231 cells. Cell Biosci. 2015;5(1):2. doi: 10.1186/20453701-5-2.

17. Rezaei-Tavirani M, Rezaei Tavirani M, Akbari Z, Hajimehdipoor $\mathrm{H}$. Prediction of coffee effects in rats with healthy and NAFLD conditions based on protein- protein interaction network analysis. Res J Pharmacogn. 2019;6(4):7-15. doi: 10.22127/rjp.2019.93500.

18. KhalKhal E, Rezaei-Tavirani M, Razzaghi MR, RezaeiTavirani S, Zali H, Rostami-Nejad M. The critical role of dysregulation of antioxidant activity and carbohydrate metabolism in celiac disease. Gastroenterol Hepatol Bed Bench. 2019;12(4):340-7. doi: 10.22037/ghfbb.v12i4.1745.

19. Zamanian-Azodi M, Rezaei-Tavirani M. Investigation of health benefits of cocoa in human colorectal cancer cell line, HT-29 through interactome analysis. Gastroenterol Hepatol Bed Bench. 2019;12(1):67-73. doi: 10.22037/ghfbb. v0i0.1557.

20. Frame MC. Src in cancer: deregulation and consequences for cell behaviour. Biochim Biophys Acta. 2002;1602(2):11430. doi: 10.1016/s0304-419x(02)00040-9.

21. Irby RB, Yeatman TJ. Role of Src expression and activation in human cancer. Oncogene. 2000;19(49):5636-42. doi: 10.1038/sj.onc.1203912.

22. Kim LC, Song L, Haura EB. Src kinases as therapeutic targets for cancer. Nat Rev Clin Oncol. 2009;6(10):587-95. doi: 10.1038/nrclinonc.2009.129.

23. Biscardi JS, Ishizawar RC, Silva CM, Parsons SJ. Tyrosine kinase signalling in breast cancer: epidermal growth factor receptor and c-Src interactions in breast cancer. Breast Cancer Res. 2000;2(3):203-10. doi: 10.1186/bcr55.

24. Liu H, Radisky DC, Nelson CM, Zhang H, Fata JE, Roth RA, et al. Mechanism of Akt1 inhibition of breast cancer cell invasion reveals a protumorigenic role for TSC2. Proc Natl Acad Sci U S A. 2006;103(11):4134-9. doi: 10.1073/ pnas.0511342103.

25. Ju X, Katiyar S, Wang C, Liu M, Jiao X, Li S, et al. Akt1 governs breast cancer progression in vivo. Proc Natl Acad Sci U S A. 2007;104(18):7438-43. doi: 10.1073/ pnas.0605874104.

26. Nicholson RI, Gee JM, Harper ME. EGFR and cancer prognosis. Eur J Cancer. 2001;37(4):S9-15. doi: 10.1016/ s0959-8049(01)00231-3.

27. Mielnicki LM, Ying AM, Head KL, Asch HL, Asch BB. Epigenetic regulation of gelsolin expression in human breast cancer cells. Exp Cell Res. 1999;249(1):161-76. doi: 10.1006/excr.1999.4461. 